\title{
Anodic Alumina Photonic Crystals as Refractive Index Sensors for Controlling the Composition of Liquid Mixtures
}

\author{
Matin ASHUROV ${ }^{1}$, Vladimir GORELIK ${ }^{2,3}$, Kirill NAPOLSKII ${ }^{1,4}$, and \\ Sergey KLIMONSKY ${ }^{1,4 *}$ \\ ${ }^{1}$ Faculty of Materials Science, Lomonosov Moscow State University, Leninskie Gory, Moscow 119991, Russia \\ ${ }^{2}$ P.N. Lebedev Physical Institute, Russian Academy of Sciences, Moscow 119991, Russia \\ ${ }^{3}$ Bauman Moscow State Technical University, Moscow 105005, Russia \\ ${ }^{4}$ Department of Chemistry, Lomonosov Moscow State University, Leninskie Gory, Moscow 119991, Russia \\ *Corresponding author: Sergey KLIMONSKY $\quad$ E-mail: klim@inorg.chem.msu.ru
}

\begin{abstract}
Photonic crystals based on anodic aluminum oxide films are examined as refractive index sensors for controlling the composition of water-alcohol liquid mixtures. The position of the reflectance maximum corresponding to the first photonic stop band is used as the analytical signal. Impregnation of a photonic crystal with water-ethanol and water-glycerol mixtures results in a redshift of the reflectance maximum. A fairly high refractive index sensitivity, sufficient to determine the composition of water-ethanol and water-glycerol mixtures with an accuracy of about $1 \mathrm{wt} . \%$, is observed. The detailed dependencies of the analytical signal on the composition of mixtures are experimentally investigated and compared with numerical calculations. Prospects and limitations of the refractive index sensors based on anodic alumina photonic crystals are discussed.
\end{abstract}

Keywords: Photonic crystal; anodic alumina; sensor; refractive index

Citation: Matin ASHUROV, Vladimir GORELIK, Kirill NAPOLSKII, and Sergey KLIMONSKY, "Anodic Alumina Photonic Crystals as Refractive Index Sensors for Controlling the Composition of Liquid Mixtures," Photonic Sensors, 2020, 10(2): 147154.

\section{Introduction}

A visually-recognizable chemical sensing or bio-sensing is a promising application of photonic crystals (PCs) [1-3]. The analytical signal may be caused by changing the effective refractive index [4-11] and the diffracting-plane spacing [12-15] (see also in [2]) or both the parameters simultaneously $[16,17]$ under the influence of certain analytes. Over the past decade, much attention has been devoted to sensors based on anodic aluminum oxide (AAO) one-dimensional PCs [18]. AAO PCs with the special functionalization can be applied to detect heavy metals, biomolecules, and drugs [19-24], whereas non-functionalized AAO PCs can be used to determine the refractive index of liquids filling the pores in their structures [23-29]. For instance, changes in the transmittance and stop band position for AAO PCs soaked in water, ethanol, ethylene glycol, and glycerol have been reported in [26]. The advantages of AAO PCs are low cost and reproducibility of fabrication process. The refractive index of aluminum oxide, $n_{\mathrm{AO}} \approx 1.76$, is high enough to work with impregnating liquids without fear of coincidence of the refractive indices and the

Received: 25 February 2019 / Revised: 26 July 2019

(C) The Author(s) 2019. This article is published with open access at Springerlink.com DOI: $10.1007 / \mathrm{s} 13320-019-0569-2$

Article type: Regular 
associated loss of the analytical signal (such situation was considered in [30] for $\mathrm{SiO}_{2} \mathrm{PCs}$ ).

However, the authors in [23, 25-27, 29] have demonstrated only the fundamental possibility of AAO PCs utilization as refractive index sensors, but have not shown the results of testing of any liquid mixtures. More detailed studies have been carried out in $[24,28]$ : the applicability of AAO PCs refractive index sensors for the detection of the composition of ethanol-isopropanol mixtures has been demonstrated [24], and linear correlation between the stop band spectral position and glucose concentration has been established [28]. Except in [28], other authors did not perform a numerical comparison of the experimental results with the expected effect of impregnating liquids on the stop band position. It is worth noting that the discrepancy between the experimental and calculated data may indicate both an incomplete filling of the pores and a wrong theoretical model of the PC structure.

Here, we demonstrate prospects and limitations of the use of anodic alumina photonic crystals for determining the composition of liquid mixtures on the examples of water-glycerol and water-ethanol solutions. Experimental results on the stop band shift are compared with numerical calculations.

\section{Materials and methods}

Anodic aluminum oxide films exhibiting the properties of one-dimensional photonic crystals were obtained by cyclic anodization as described in [31, 32]. High-purity aluminum foil (99.99\%, $0.1 \mathrm{~mm}$ thickness) with an electropolished surface was used as the starting material. The anodization was carried out in $1.0 \mathrm{M}$ sulfuric acid electrolyte under square-wave current oscillation. The anodization area $\left(5.7 \mathrm{~cm}^{2}\right)$ was restricted by o-ring. The electrolyte was continuously stirred and the environment temperature was kept constant at $(2 \pm 1){ }^{\circ} \mathrm{C}$. The initial charge of the low current stage $(2.2 \mathrm{~mA})$ and high current stage $(12.3 \mathrm{~mA})$ was
$1.22 \mathrm{C}$, which corresponded to the duration of $555 \mathrm{~s}$ and $100 \mathrm{~s}$ for the low-current stage and high-current stage, respectively. The charge of each subsequent anodizing cycle was reduced by $0.1 \%$ to compensate for the effect of chemical etching of upper porous oxide layers in the acidic electrolyte. The scanning electron microscopy image of a sample cross section is shown in Fig. 1. The structure consists of alternating layers with different porosities. Optical properties of AAO PCs films obtained under similar conditions were described previously in $[32,33]$.

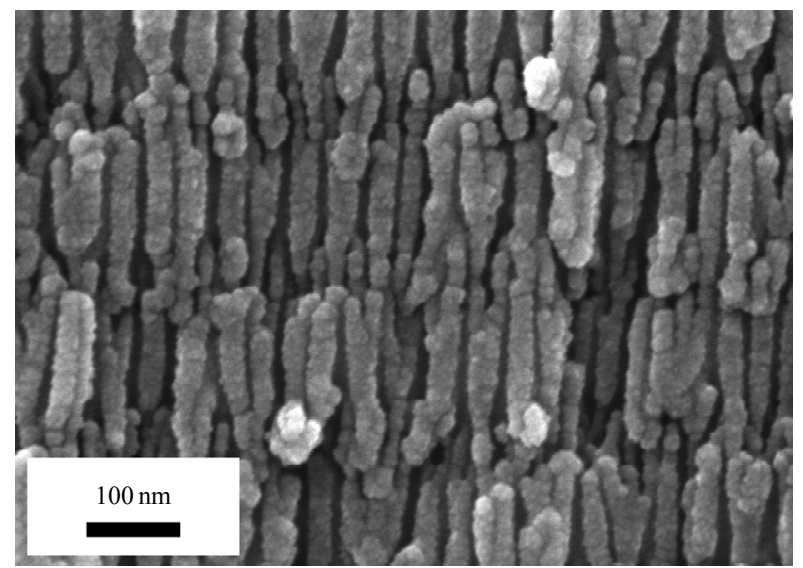

Fig. 1 Scanning electron microscopy image of the AAO PC cross section.

The samples were impregnated with liquids inside a closed plastic vessel completely isolated from the environment. The local reflection spectra were recorded at close-to-normal incidence of light on the AAO surface at the room temperature $\left(20^{\circ} \mathrm{C}\right)$ using a halogen lamp as the broadband light source (working spectral range of $400 \mathrm{~nm}-900 \mathrm{~nm}$ ), the $100-\mu \mathrm{m}$ quartz optical fibers, and the FSD8 spectrometer with a working spectral range of $200 \mathrm{~nm}-1000 \mathrm{~nm}$ and a registration step of about $0.25 \mathrm{~nm}$. The measurement scheme with a vertical light probe (see the inset in Fig. 2) was similar to that described in [5], but we recorded the spectra through the bottom of the vessel. In the case of the absence of AAO PC sample, the reflectance of the bottom of the vessel was less than $2 \%$ without sharp variations in its spectral dependence at wavelengths of exceeding $510 \mathrm{~nm}$. 


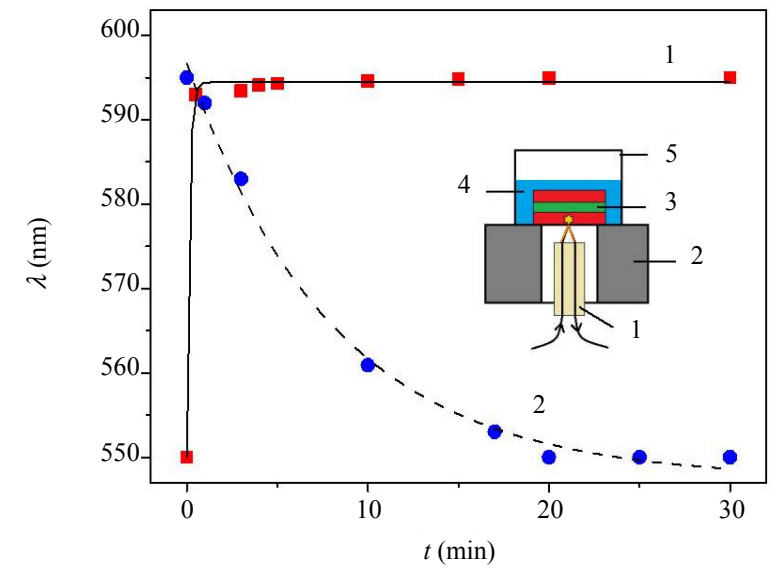

Fig. 2 Time dependencies of the wavelength of the first stop band of AAO PC during impregnation with water-ethanol (42.5 wt. \%) mixture (squares) and subsequent drying (circles). The size of the symbols corresponds to the size of error bars. Lines 1 and 2 are given just for eye guide. The measurement scheme $(1$ - optical fiber probe; 2 - mounting platform; 3 sample; 4 - analyte; 5 - plastic vessel) is given in inset.

The recorded spectra for AAO PCs were averaged over five different points of the sample. To obtain the spectral dependence of the sample reflectance, the averaged spectrum of the reflected light was divided into the same spectrum obtained for a silver mirror PF10-03-P01 (THORLABS), whose reflectance in the visible range was $(99 \pm 1) \%$. We did not analyze the height of the reflectance peak connected with the first order photonic stop band because of its point-to-point variations observed in local measurements [33]. In contrast, the wavelength of the peak was a much more stable parameter. The reflectance peak was approximated by a cubic function for determination of its position with an accuracy of the order of $0.1 \mathrm{~nm}$.

The transmittance in the normal direction and the specular reflectance in the angular range from $8^{\circ}$ to $65^{\circ}$ were recorded with a Lambda 950 spectrophotometer (Perkin Elmer).

In order to study the performance of AAO PCs optical sensors, glycerol ( $\geq 99.7 \%$, Oleon), ethanol ( $\geq 99.9 \%$, Himsintez), and their mixtures with deionized water were used as analytes. Impregnation of the samples with water-ethanol mixtures and further drying were carried out at a temperature of $70{ }^{\circ} \mathrm{C}$ by using drying oven FED 53 (Binder). The time-evolution of the spectral position of the reflectance peak during impregnation with 42.5 wt. \% ethanol mixture and further drying are shown in Fig. 2. It can be seen that the impregnation leads to a redshift of the photonic stop band position, whereas the drying results in its recovering. Both processes are completed after 20 minutes. Accordingly, the duration of the impregnation and drying was chosen equal to 20 minutes in further experiments with water-ethanol mixtures. However, such process was not sufficient to impregnate with glycerol due to its high viscosity (see Fig. 3). In order to reach the complete impregnation of the samples with water-glycerol mixtures, 5 days -7 days of soaking in corresponding liquid at the room temperature were added to 20 minutes of soaking at $70^{\circ} \mathrm{C}$.

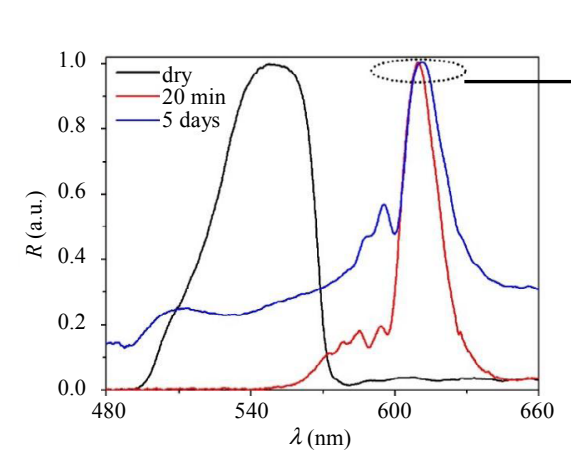

(a)

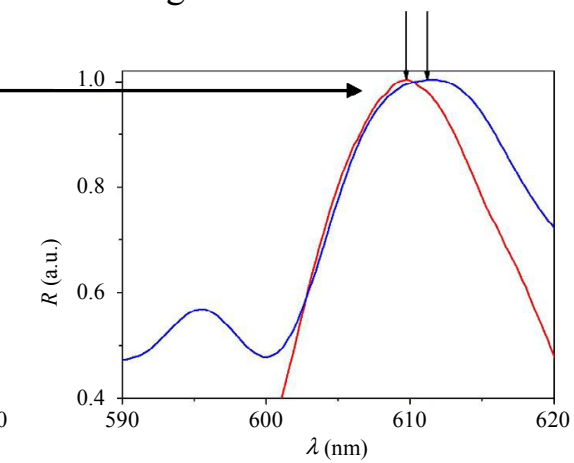

(b)

Fig. 3 Results of glycerol impregnation: (a) normalized reflectance spectra of 1D PhC based on AAO in the dry state (black line), after 20 minutes impregnation with glycerol at $70{ }^{\circ} \mathrm{C}$ (red line) and after additional impregnation with glycerol for 5 days at room temperature (blue line) and (b) reflectance peaks after different impregnation methods, shown in close-up. The spectral positions of the maxima ( $609.5 \mathrm{~nm}$ and $611.4 \mathrm{~nm}$ for red and blue curves, correspondingly) are indicated by arrows. 


\section{Results and discussion}

Photonic crystals based on anodic aluminum oxide films demonstrate clear stop band shift with the impregnation by different liquids. Figure 4 shows examples of normalized reflectance spectra of the AAO PC sample infiltrated by water, glycerol, and their mixture with $46 \mathrm{wt} . \%$ of glycerol. Detailed data for the reflectance peak position for water-glycerol and water-ethanol mixtures are given in Fig. 5(a) in comparison with the calculations based on Bragg diffraction formula:

$$
2 d n_{\text {eff }}=\lambda \text {. }
$$

The corresponding data for full width at half maximum (FWHM) of the peak are shown in Fig. 5(b). Here, $d$ is the structure period of the photonic crystal, $\lambda$ is the light wavelength in vacuum,

$$
n_{\mathrm{eff}}=\sqrt{n_{i}^{2} P+n_{\mathrm{AO}}^{2}(1-P)}
$$

is the effective refractive index of the porous structure, $n_{i}$ is a refractive index of the media inside the pores (air or infiltrating liquid), $n_{\mathrm{AO}}=1.76$ is the refractive index of aluminum oxide, and $P$ is the porosity averaged over alternating layers. Equation (2) is valid if we neglect the depolarization factor of the pores. Reference data for refractive indices $n_{i}$ were taken from [34], and other parameters used in calculations were $d=186.1 \mathrm{~nm}$ and $P=0.435$.

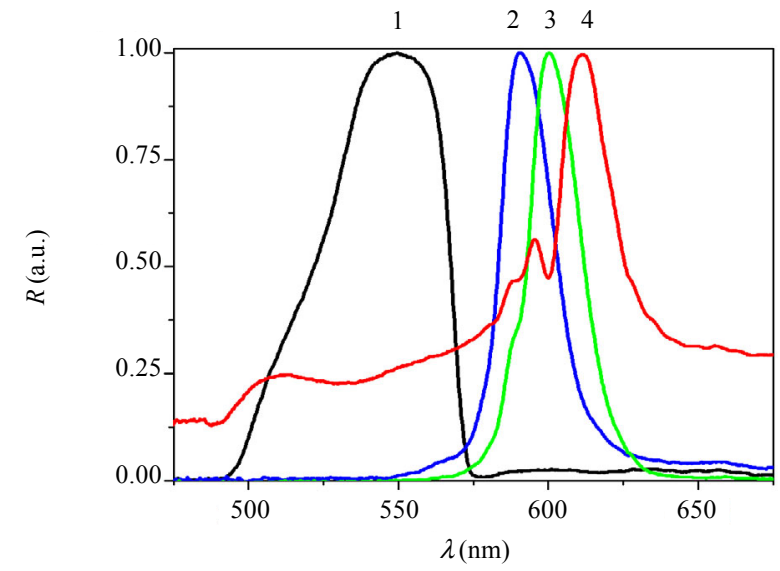

Fig. 4 Normalized reflection spectra for the AAO PC sample in dry state (1) and immersed in various liquids: water (2), water with 46 wt.\% glycerol (3), and glycerol (4).
As can be seen from Fig. 5(a), non-monotonic dependence was obtained for water-ethanol mixtures because of non-monotonic changes in their refractive indices [34], whereas for water-glycerol mixtures it was almost linear. All experimental data were in good agreement with calculated curves; the valid peak position $\lambda=549.6 \mathrm{~nm}$ for dry sample (with air in channels, $n_{i}=1$ ) was obtained with the same parameters too. Thus, photonic crystals based on anodic aluminum oxide could be used for sensing of refractive index and composition of various liquid mixtures.

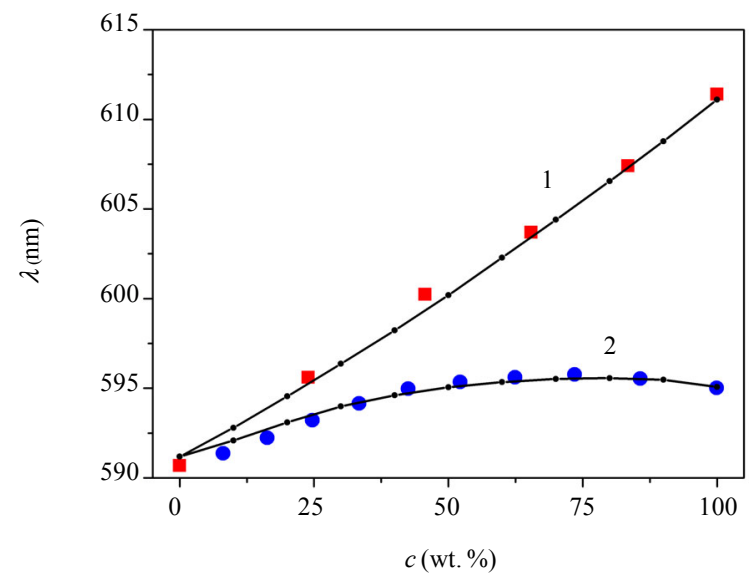

(a)

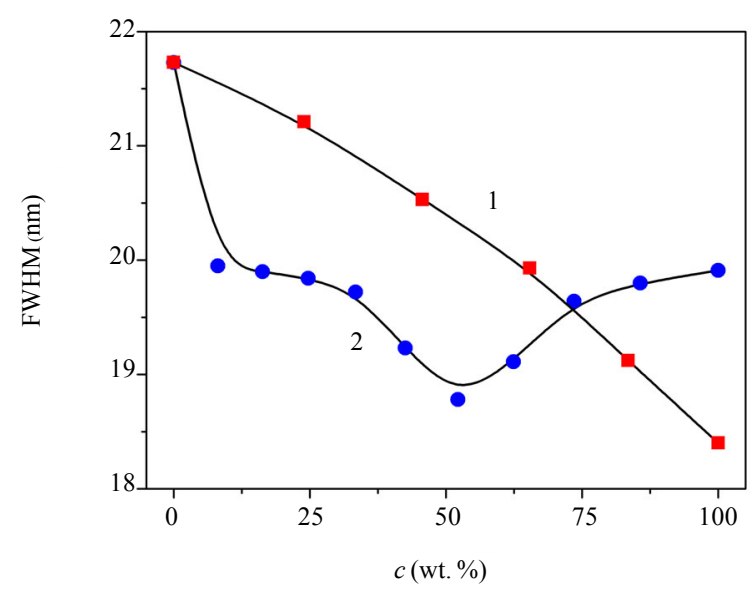

(b)

Fig. 5 Dependence of the wavelength (a) and the width (b) of the first stop band of AAO PC on the composition of immersion liquid. Experimental data for water-glycerol (1) and water-ethanol (2) mixtures are shown by squares and circles, respectively. The size of the symbols corresponds to the size of error bars. Black dots connected by lines in (a) are calculated data in accordance with (1) and (2) using reference data on the refractive indices [34]. Solid lines in (b) are given just for eye guide. 
In accordance with Figs. 4 and 5(a), the reflectance peaks locate at $591 \mathrm{~nm}$ for water $\left(n_{i}=\right.$ $1.333)$ and at $611 \mathrm{~nm}$ for glycerol $\left(n_{i}=1.474\right)$. Based on these data, we could calculate the refractive index sensitivity of our samples $\Delta \lambda / \Delta n=142 \mathrm{~nm} / \mathrm{RIU}$. This value is greater than ones reported in $[25,26]$ (see Table 1) and comparable with that in [28], but lower than the sensitivity demonstrated in [27] by using the graded-lattice AAO PCs (441 nm/RIU) and the one obtained in [29] by using apodized samples $(390 \mathrm{~nm} / \mathrm{RIU})$. An additional chemical etching was applied to enhance the porosity and corresponding volume fraction of analyzed liquid in $[27,29]$. The porosity of our samples was about 44 vol.\%. We could increase the porosity $P$ and enhance corresponding refractive index sensitivity at least twice by additional etching too, but samples became very fragile in this case. On the other hand, it is obvious from Fig. 5(a) that even without such etching, the achieved sensitivity of 142 $\mathrm{nm} / \mathrm{RIU}$ was enough for the detection of the composition of water-ethanol and water-glycerol mixtures with the accuracy of about $1 \mathrm{wt} . \%$.

Table 1 Refractive index sensitivities of AAO PCs reported in the literatures.

\begin{tabular}{ccc}
\hline $\begin{array}{c}\text { Preparation technique of } \\
\text { AAO PCs }\end{array}$ & $\begin{array}{c}\text { Refractive index } \\
\text { sensitivity (nm/RIU) }\end{array}$ & Reference \\
\hline $\begin{array}{c}\text { Periodical modulation of } \\
\text { current density } \\
\text { Sine-wave current of } \\
\text { anodization }\end{array}$ & 71 & {$[25]$} \\
$\begin{array}{c}\text { Multistep pseudosinusoidal } \\
\text { voltage modulation }\end{array}$ & 109 & {$[26]$} \\
$\begin{array}{c}\text { Graded-lattice anodization } \\
\text { with additional chemical } \\
\text { etching }\end{array}$ & 164 & {$[28]$} \\
Apodization strategy with \\
additional chemical etching \\
Square-wave current \\
oscillation
\end{tabular}

The FWHM of the reflectance peak [Fig. 5(b)] decreases with an increase in the refractive index of water-glycerol mixtures due to the drop in the optical contrast of the impregnated sample. For water-ethanol mixtures, a non-monotonic character of the dependence is similar to that in Fig. 5(a). However, the full correlation of the FWHM with the refractive index is missing (liquids with different refractive indices may give the same peak width), so this parameter, as well as the peak height, is not suitable for sensing. It is worth noting that the peak width is highly sensitive to the structure imperfection and inhomogeneity. The mismatch of the stop bands for the front and back sides of AAO PC should also be taken into account [33]. Such mismatch can lead to not only the broadening of the reflectance peak, but also the appearance of an additional maximum on its left side, as shown by Curve 4 in Fig. 4.

The data shown in Fig. 5 were obtained for the same sample repeatedly impregnated with different liquids, and the good reproducibility in the spectral position of the stop band was observed. After the last impregnation with $24 \mathrm{wt} . \%$ glycerol solution, the sample was kept in a dry state for 2 months. Then, additional measurements were undertaken for reproducibility control. It was established that the optical properties of the sample changed: the stop band shifted by about $5 \mathrm{~nm}$ to longer wavelengths, both in the dry state and with water.

It is known that AAO produced in a highly concentrated sulfuric acid electrolyte has a low chemical stability [35] and undergoes structural alterations when heated in water-based solutions [36]. So we decided to test the stability of our samples. To do this, we took a new sample and carried out with it 10 cycles, consisting of water impregnation during 20 minutes at $70{ }^{\circ} \mathrm{C}$ and further drying during 20 minutes at the same temperature. Corresponding changes in the transmittance spectrum are shown in Fig. 6.

It can be seen that after 4 cycles, the changes in the spectrum were negligible, and the position of the spectrum minimum was unchanged. However, after 10 cycles of the impregnation and drying, the 
redshift of the minimum of about $5 \mathrm{~nm}$ appeared (from $555 \mathrm{~nm}$ to $560 \mathrm{~nm}$ ). Even greater shift of about $7 \mathrm{~nm}$ (from $554 \mathrm{~nm}$ to $561 \mathrm{~nm}$ ) was seen for the reflectance peak (see the inset in Fig. 6). It means that the effective refractive index became higher. On the other hand, the period of the Fabry-Perot fringes observed in the long wavelength region of both transmittance and reflectance spectra (Fig. 6) was reduced by about $1 \%$. This fact points out that some materials were washed out, so a decrease in the effective refractive index averaged over the entire sample thickness was observed. Such contradictive results can be explained if we assume that during repeated impregnation of pores by water and drying, some species were redistributed from the bottom part of AAO PC film to the sample volume and/or partially removed from the porous structure by the water flow. In such a case, the averaged refractive index would decrease, whereas the effective refractive index of the upper layers, forming the reflectance peak, would increase. Thus, the sample stability is an essential limitation in the present state of the technology. The degradation of anodic alumina in pure water can be caused by the dissolution of water-soluble $\left[\mathrm{Al}_{13} \mathrm{O}_{4}(\mathrm{OH})_{24}\left(\mathrm{H}_{2} \mathrm{O}\right)_{12}\right]^{7+}$ polyhydroxocomplexes and their further redeposition in the form of $\left[\mathrm{Al}(\mathrm{OH})_{4}\right]^{-}[37]$. In order to improve the chemical stability of porous anodic

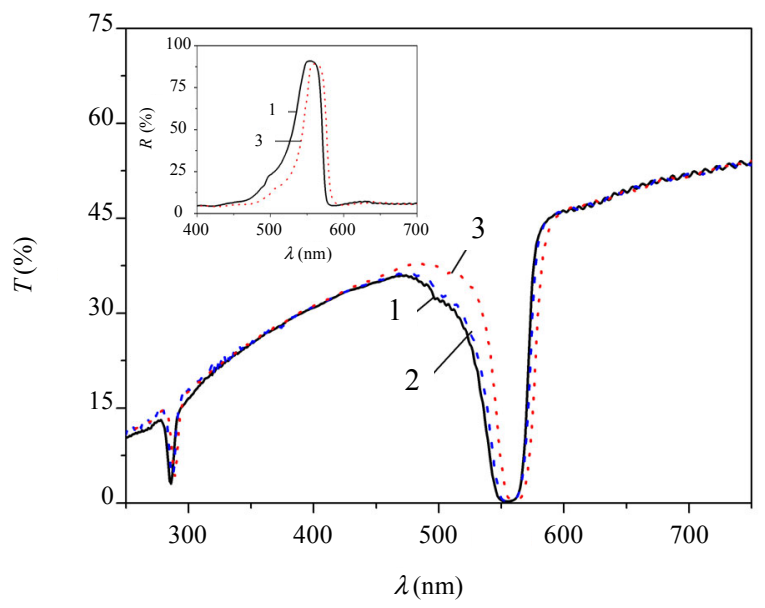

Fig. 6 Transmittance and reflectance (see the inset) spectra of initial AAO PC (1) and the same sample after 4 (2) cycles and 10 (3) cycles of impregnation and drying. alumina, various approaches have been suggested, such as the modification of the pore walls [37] and the crystallization of initially amorphous AAO at high temperatures [38].

\section{Conclusions}

It has been shown that the impregnation of photonic crystals based on anodic aluminum oxide films with water-ethanol and water-glycerol mixtures results in a substantial redshift of the reflectance maximum, which can be calculated with the same PC parameters for all cases without taking into account the depolarization factor of the structural pores. A fairly high refractive index sensitivity $\Delta \lambda / \Delta n=142 \mathrm{~nm} / \mathrm{RIU}$ has been achieved. The possibility of precise control of the composition of liquid mixtures using photonic crystal films has been demonstrated. Such control can be very fast in the case of water-ethanol mixtures, whereas in the case of highly viscous liquids, such as glycerol, soaking AAO PCs for several days for complete infiltration is required. Besides that, a long-term practical application of AAO PCs as refractive index sensors requires that the problem of the stability of the samples with respect to multiple water impregnation should be solved.

\section{Acknowledgment}

The work was partially supported by the Russian Foundation for Basic Research (Grant No. 18-0200181).

Open Access This article is distributed under the terms of the Creative Commons Attribution 4.0 International License (http://creativecommons.org/licenses/by/4.0/), which permits unrestricted use, distribution, and reproduction in any medium, provided you give appropriate credit to the original author(s) and the source, provide a link to the Creative Commons license, and indicate if changes were made.

\section{References}

[1] J. F. Galisteo-Lopez, M. Ibisate, R. Sapienza, L. S. 
Froufe-Perez, A. Blanco, and C. Lopez, "Self-assembled photonic structures," Advanced Materials, 2011, 23(1): 30-69.

[2] Y. Zhao, Z. Xie, H. Gu, C. Zhu, and Z. Gu, "Bio-inspired variable structural color materials," Chemical Society Reviews, 2012, 41(8): 3297-3317.

[3] H. Wang and K. Q. Zhang, "Photonic crystal structures with tunable structure color as colorimetric sensors," Sensors, 2013, 13(4): 41924213.

[4] C. Y. Kuo, S. Y. Lu, S. F. Chen, M. Bernards, and S. Y. Jiang, "Stop band shift based chemical sensing with three-dimensional opal and inverse opal structures," Sensors and Actuators B: Chemical, 2007, 124(2): 452-458.

[5] J. L. Li and T. S. Zheng, "A comparison of chemical sensors based on the different ordered inverse opal films," Sensors and Actuators B: Chemical, 2008, 131(1): 190-195.

[6] W. K. Kuo, H. P. Weng, J. J. Hsu, and H. H. Yu, "Photonic crystal-based sensors for detecting alcohol concentration," Applied Sciences, 2016, 6(3): 67.

[7] S. Amrehn, X. Wu, C. Schumacher, and T. Wagner, "Photonic crystal-based fluid sensors: toward practical application," Physica Status Solidi (A), 2015, 212(6): 1266-1272.

[8] M. Nejadebrahimy, L. Halimi, and $\mathrm{H}$. Alipour-Banaei, "Design and simulation of ultrasensitive nano-biosensor based on OFPC," Photonic Sensors, 2015, 5(1): 43-49.

[9] S. Sahu, J. Ali, P. P. Yupapin, and G. Singh, "Porous silicon based Bragg-grating resonator for refractive index biosensor," Photonic Sensors, 2018, 8(3): 248-254.

[10] R. Arunkumar, T. Suaganya, and S. Robinson, "Design and analysis of 2D photonic crystal based biosensor to detect different blood components," Photonic Sensors, 2019, 9(1): 69-77.

[11] Y. Wang, W. Cheng, J. Qin, and Z. Han, "Terahertz refractive index sensor based on the guided resonance in a photonic crystal slab," Optics Communications, 2019, 434: 163-166.

[12] J. H. Holtz and S. A. Asher, "Polymerized colloidal crystal hydrogel films as intelligent chemical sensing materials," Nature, 1997, 389(6653): 829-832.

[13] Y. J. Zhao, X. W. Zhao, B. C. Tang, W. Y. Xu, J. Li, L. $\mathrm{Hu}$, et al., "Quantum-dot-tagged bioresponsive hydrogel suspension array for multiplex label-free DNA detection," Advanced Functional Materials, 2010, 20(6): 976-982.

[14] Z. Cai, N. L. Smith, J. T. Zhang, and S. A. Asher, "Two-dimensional photonic crystal chemical and biomolecular sensors," Analytical Chemistry, 2015, 87(10): 5013-5025.

[15] Z. Cai, A. Sasmal, X. Liu, and S. A. Asher, "Responsive photonic crystal carbohydrate hydrogel sensor materials for selective and sensitive lectin protein detection," ACS Sensors, 2017, 2(10): 14741481.

[16] F. Wang, Z. Zhu, M. Xue, F. Xue, Q. Wang, Z. Meng, et al., "Cellulose photonic crystal film sensor for alcohols," Sensors and Actuators B: Chemical, 2015, 220: 222-226.

[17] D. Kou, S. Zhang, J. L. Lutkenhaus, L. Wang, B. Tang, and W. Ma, "Porous organic/inorganic hybrid one-dimensional photonic crystals for rapid visual detection of organic solvents," Journal of Materials Chemistry C, 2018, 6(11): 2704-2711.

[18] C. S. Law, S. Y. Lim, A. D. Abell, N. H. Voelcker, and A. Santos, "Nanoporous anodic alumina photonic crystals for optical chemo-and biosensing: fundamentals, advances, and perspectives," Nanomaterials, 2018, 8(10): 788.

[19] Y. Chen, A. Santos, Y Wang, T. Kumeria, D. Ho, J. Li, et al., "Rational design of photonic dust from nanoporous anodic alumina films: a versatile photonic nanotool for visual sensing," Scientific Reports, 2015, 5: 12893.

[20] A. Santos, J. H Yoo, C. V. Rohatgi, T. Kumeria, Y Wang, and D. Losic, "Realisation and advanced engineering of true optical rugate filters based on nanoporous anodic alumina by sinusoidal pulse anodisation," Nanoscale, 2016, 8(3): 1360-1373.

[21] C. S. Law, S. Y. Lim, A. D. Abell, and A. Santos, "Real-time binding monitoring between human blood proteins and heavy metal ions in nanoporous anodic alumina photonic crystals," Analytical Chemistry, 2018, 90(16): 10039-10048.

[22] M. Nemati, A. Santos, and D. Losic, "Fabrication and optimization of bilayered nanoporous anodic alumina structures as multi-point interferometric sensing platform," Sensors, 2018, 18(2): 470.

[23] Y. Chen, A. Santos, Y. Wang, T. Kumeria, C. Wang, J. Li, et al., "Interferometric nanoporous anodic alumina photonic coatings for optical sensing," Nanoscale, 2015, 7(17): 7770-7779.

[24] Y. Chen, A. Santos, Y. Wang, T. Kumeria, J. Li, C. Wang, et al., "Biomimetic nanoporous anodic alumina distributed bragg reflectors in the form of films and microsized particles for sensing applications," ACS Applied Materials \& Interfaces, 2015, 7(35): 19816-19824.

[25] D. L. Guo, L. X. Fan, F. H. Wang, S. Y. Huang, and $\mathrm{X}$. W. Zou, "Porous anodic aluminum oxide Bragg stacks as chemical sensors," The Journal of Physical Chemistry C, 2008, 112(46): 17952-17956.

[26] P. Yan, G. T. Fei, G. L. Shang, B. Wu, and L. D. Zhang, "Fabrication of one-dimensional alumina photonic crystals with a narrow band gap 
and their application to high-sensitivity sensors," Journal of Materials Chemistry C, 2013, 1(8): 16591664.

[27] J. Lee, K. Bae, G. Kang, M. Choi, S. Baek, D. Yoo, et al., "Graded-lattice AAO photonic crystal heterostructure for high Q refractive index sensing," RSC Advances, 2015, 5(88): 71770-71777.

[28] T. Kumeria, M. M. Rahman, A. Santos, J. Ferre-Borrull, L. F. Marsal, and D. Losik, "Structural and optical nanoengineering of nanoporous anodic alumina rugate filters for real-time and label-free biosensing applications," Analytical Chemistry, 2014, 86(3): 18371844.

[29] C. S. Law, S. Y. Lim, and A. Santos, "On the precise tuning of optical filtering features in nanoporous anodic alumina distributed Bragg reflectors," Scientific Reports, 2018, 8(1): 4642.

[30] M. V. Vasnetsov, T. N. Orlova, V. Y. Bazhenov, A. S. Shevchuk, A. D. Kudryavtseva, and N. V. Tcherniega, "Photonic bandgap examination in an immersed synthetic opal," Applied Physics B, 2014, 116(3): 541-548.

[31] L. Yisen, C. Yi, L. Zhiyuan, H. Xing, and L. Yi, "Structural coloring of aluminum," Electrochemistry Communications, 2011, 13(12): 1336-1339.

[32] S. E. Kushnir and K. S. Napolskii, "Thicknessdependent iridescence of one-dimensional photonic crystals based on anodic alumina," Materials \& Design, 2018, 144: 140-150.

[33] V. S. Gorelik, S. O. Klimonsky, V. V. Filatov, and K. S. Napolskii, "Optical properties of one-dimensional photonic crystals based on porous films of anodic aluminum oxide," Optics and Spectroscopy, 2016, 120(4): 534-539.

[34] CRC handbook of chemistry and physics: Section 8: Analytical Chemistry, 84th ed., D.R. Lide (Editor). Boca Raton, Florida, USA: CRC Press, 2004: $62-65$.

[35] T. Yanagishita and H. Masuda, "Facile preparation of porous alumina through-hole masks for sputtering by two-layer anodization," AIP Advances, 2016, 6(8): 085108.

[36] D. Mattia and H. Leese, "Controlled hydrothermal pore reduction in anodic alumina membranes," Nanoscale, 2014, 6(22): 13952-13957.

[37] D. I. Petukhov, D. A. Buldakov, A. A. Tishkin, A. V. Lukashin, and A. A. Eliseev, "Liquid permeation and chemical stability of anodic alumina membranes," Beilstein Journal of Nanotechnology, 2017, 8(1): 561-570.

[38] A. P. Leontiev, O. A. Brylev, and K. S. Napolskii, "Arrays of rhodium nanowires based on anodic alumina: preparation and electrocatalytic activity for nitrate reduction," Electrochimica Acta, 2015, 155: 466-473. 